\title{
Erratum: Quantum mechanical prediction of four-phonon scattering rates and reduced thermal conductivity of solids [Phys. Rev. B 93, 045202 (2016)]
}

Tianli Feng and Xiulin Ruan

Q (Received 25 January 2018; published 5 February 2018)

DOI: 10.1103/PhysRevB.97.079901

Equation (33) and its description have some errors:

(1) The factor "96 * 2" in Eq. (33) should read "96 *6."

(2) On p. 4, the text "In both Eqs. (31) and (33), the factor 2 in front of $\frac{2 \pi}{\hbar}$ accounts for the difference between scattering rate and self-energy linewidth." should read "The factor 2 in front of $\frac{2 \pi}{\hbar}$ in Eq. (31) accounts for the permutation of $\lambda_{1}$ and $\lambda_{2}$. Similarly, the factor 6 in front of $\frac{2 \pi}{\hbar}$ in Eq. (33) accounts for the permutation of $\lambda_{1}, \lambda_{2}$, and $\lambda_{3}$."

The other equations and all the results are unaffected.

The last sentence in the caption of Fig. 3 is not precise enough. It should read "The phonon dispersion used in the calculation of $\kappa_{\mathrm{NMA}}$ (where NMA represents normal mode analysis) is from the lattice dynamics (LD) calculation with the lattice constant at $0 \mathrm{~K}$ to be consistent with the $\kappa_{3}$ and $\kappa_{3+4}$ calculations." 